\title{
VI.
}

\section{Die Bedeutung des Molecularzustandes der wassergelösten Desinfectionsmittel für ihren Wirkungswerth.}

\author{
Von \\ Stabsarzt Dr. Scheurlen, \\ Privatdozent f. Hygiene u. Bacteriologie an der Universitāt Strassburg.
}

Der Wirkungswerth unserer Desinfectionsmittel ist um so grösser, je concentrirter ihre Lösung und je höher deren Temperatur ist. Das sind zwei durch zahlreiche Untersuchungen zu bacteriologischen Grundbegriffen gewordene Facta, dass es fast scheint, als ob uber der Selbstverständlichkeit die Frage nach der Ursache dieser doch keineswegs so ohne Weiteres erklärlichen Erscheinung vergessen worden wäre.

Ich gehe von dem Beispiel aus, dass eine 6 proc. wässrige Carbollösung, bei Zimmertemperatur, also etwa $20^{\circ} \mathrm{C}$., Milzbrandsporen in ungefähr 8 Tagen vernichtet, was eine 3 proc. bei dieser Temperatur uberhaupt nicht oder jedenfalls erst in viel längerer Zeit zu leisten vermag; erwärmt man aber letztere auf $40-45^{\circ} \mathrm{C}$., so erhält sie dieselbe Desinfectionskraft wie jene. Dass es sich in diesem Falle nicht um die gemeinsame Action zweier gleichzeitig unmittelbar auf die Milzbrandsporen wirkender Desinficientien, des Phenols und der Wärme handelt, welch letztere man wohl früher als besonderes physikalisches Desinfectionsmittel angesehen hat, erhellt daraus, dass $40-45^{\circ}$ in Luft oder Wasser den Milzbrandsporen nichts anzuhaben vermögen.

Es spitzt sich also unsere Frage dahin zu: was für eine gemeinsame Eigenschaft besitzt das Phenolmolecill in der gesättigten wässrigen Lösung und in der nicht gesättigten aber erwärmten Lösung? Oder allgemeiner gefasst: in was fir einem Zustand befindet sich das Phenolmolecuil überhaupt in wässriger Lösung?

Zur Beantwortung dieser Frage milssen wir etwas weit ausholen.

Der Zustand der Molecüle in Lösungen ist bis jetzt am eifrigsten an Salzen studirt worden. Die diesbezüglichen Untersuchungen 
sind nicht einfach, denn die Körper selbst können nicht zur unmittelbaren Untersuchung herangezogen werden, da die Beschaffenheit eines aus Wasser auskrystallisirten, z. B. bydrathaltigen Salzes keine Gewähr bietet, dass das Salz in dieser Zusammensetzung sich in der Lösung befunden hat.

Aufschluss haben hier gegeben die Untersuchungen uber die Erniedrigung des Gefrierpunktes, des Dichtemaximums und der Spannkraft (Erhöhung des Siedepunktes); ferner das Verhalten der Lösungswärme hydrathaltiger Salze gegentiber derjenigen ihrer Anhydrite; weiterhin die plötzliche Veränderung der Löslichkeitscurve eines Salzes, wie z. B. des Glaubersalzes und der Soda, und schliesslich das Verhalten verschiedener farbiger Salze, deren Farbe sich bei Anfnahme von Hydratwasser verändert.

Aus diesen Untersuchungen hat sich für die weitaus iiberwiegende Mehrzahl der Salze das sichere Resultat ergeben, dass ibre Molecüle sich als Hydrate in der wässrigen Lösung befinden.

Ich gehe nicht auf alle diese Untersuchungen näher ein, sie können in dem bekannten Lehrbuch von 0 stwald nachgelesen werden; ich erwähne nur kurz zur Orientirung für diejenigen, denen die einschlägigen Verhältnisse nicht geläufig sind, als Beispiel: die Untersuchungen ubber die Gefrierpunktserniedrigung und über das Verhalten einiger hydrathaltiger farbiger Salze; letztere hebe ich besonders deshalb hervor, da von ihnen in der Hauptsache meine Untersuchungen ihren Ausgang genommen haben.

Wenn man zu $100 \mathrm{~g}$ Wasser eine bestimmte Menge Kochsalz hinzusetzt, so erniedrigt sich der Gefrierpunkt des Wassers um eine bestimmte Grösse, die proportional ist der zugefiugten Kochsalzmenge; und zwar ist die Erniedrigung (E), dividirt durch die Menge Salz in $100 \mathrm{~g}$ Wasser (M), eine constante Grösse, der sogenannte Erniedrigungscoefficient: $\mathrm{E} / \mathrm{M}=$ const. Nun bleibt z. B. beim Kochsalz der Erniedrigungscoefficient constant bis zu einer Erniedrigung des Gefrierpunktes auf $-9^{0}$, was einem Gehalt von 15 Proc. Kochsalz entspricht, bei weiterem Zusatz nimmt der Quotient $\mathrm{E} / \mathrm{M} \mathrm{zu}$, giebt aber sofort wieder eine constante Grösse, wenn man das Kochsalz mit 2 Molecülen Krystallwasser verbunden in Rechnung zieht. Noch markanter erscheinen diese Verhältnisse bei dem später zu erwähnenden Kupferchlorid. Dasselbe giebt bis zu einem Gehalt von 16 Proc. und einer Temperaturerniedrigung auf $-7,5^{\circ} \mathrm{C}$. unter Annahme von 12 Molecillen Hydratwasser einen constanten Erniedrigungscoefficienten, verlangt aber bei weiterer Concentration und Temperaturerniedrigung die Annabme von 4 Molecullen Krystallwasser. Gleichzeitig 
findet hier als weiterer Beweis für die thatsächlich sich ändernden Hydratverhältnisse die sofort $\mathbf{z u}$ besprechende Farbenveränderung von blau in grün statt.

Das Kupferchlorid krystallisirt mit 2 Molecülen Krystallwasser in grünen rhombischen Krystallen. Starke wässrige Lösungen dieses Salzes sehen gleichfalls grün aus und enthalten, wie oben gesagt, ein 4 Molecüle Hydratwasser enthaltendes Kupferchlorid, während schwächere, z. B. 10 proc. Lösungen, eine blaue Farbe zeigen und, wie aus. den erwähnten Untersuchungen über die Gefrierpunktserniedrigung hervorgegangen ist, das Kupferchlorid mit 12 Molecülen Krystallwasser verbunden enthalten. Erwärmt man diese Lösung auf $75^{\circ} \mathrm{C}$., so ist sie bei $75^{\circ} \mathrm{C}$. rein grün, abgekublt erscheint sie bei $50^{\circ} \mathrm{C}$. wieder rein blau, ${ }^{1}$ )

Setzt man zu $10 \mathrm{ccm}$ der 10 proc. Lösung bei $20^{\circ} \mathrm{C} .2 \mathrm{~g}$ Kochsalz, so wird sie bei dieser Temperatur grtin, das Gleiche geschieht, wenn $2 \mathrm{~g}$ Calciumchlorid zugesetzt werden, wobei ausserdem durch theilweise ausgefälltes Kupferchlorid ein grüner Niederschlag entsteht.

Das Kobaltchlorür, die sympathetische Tinte, krystallisirt mit 6 Molectilen Krystallwasser in schönen rothen monosymmetrischen Krystallen. Erhitzt schmelzen dieselben in ihrem Krystallwasser, geben es dann ab und es bleibt eine amorphe Masse von blauer Farbe: das wasserfreie Kobaltchlorür.

In Wasser gelöst, bildet das Cobaltum chloratum purum crystallisatum, mit dem ich experimentirte, eine rosarothe Flussigkeit, die in verdtinnter Lösung zum Kochen erhitzt, sich in der Farbe nicht verändert; nimmt man eine stärkere, 25 proc. Lösung, so erhält dieselbe beim Kochen nur einen Stich ins Bläuliche, nimmt man eine 75 proc. Lösung, so wird diese, zum Kochen erhitzt, tiefblau. Fügt man nun zu $10 \mathrm{ccm}$ der 25 proc. Lösung $5 \mathrm{~g}$ Kochsalz hinzu, so wird diese schon bei $50^{\circ} \mathrm{C}$. tiefblau, ohne dass sich übrigens alles Kochsalz lösen würde. Setzt man zur selben Menge derselben Lösung $2 \mathrm{~g}$ Calciumchlorid, so wird die Lösung bei $70^{\circ} \mathrm{C}$. tiefblau, gleichzeitig. tritt auch hier eine Ausfällung des blauen Kobaltchloriars ein. Fỉgt man dagegen $\mathrm{zu} 10 \mathrm{ccm}$ der 25 proc. Lösung $10 \mathrm{~g}$ Chlorzink, so verändert sich die rothe Farbe beim Kochen nicht, bezw. nicht mehr als ohne Chlorzinkzusatz.

Gestiutzt auf die oben angeftuhrten Resultate anderer Untersuch-

1) Diese Zablen sind keine Grenzwerthe, welche auf diese Weise zu erhalten überhaupt nicht möglich ist, da durch verschiedene Zwischenfarben grün in blau übergeht. 
ungen geht aus diesen ïbrigens keineswegs neuen Versuchen hervor, dass die Salze in concentrirter wässriger Lösung wenig oder gar kein Hydratwasser enthalten, dass sie aber in verdünter Lösung mehr oder weniger reichlich damit verbunden sind. Dieses Hydratwasser verlieren sie sowohl durch Erwärmen als durch Zusatz stärker wasseranziehender Substanzen, so dass sie schliesslich in demjenigen Molecularzustand in der Lösung sich befinden, den sie, wie z. B. das Kobaltchlorür, iu ihrer concentrirtesten, also wohl auch wirksamsten Form einnehmen.

Die Versuche baben weiterhin ergeben, dass die Salze sich in ibrer wasserentziehenden Wirkung nicht gleichwerthig verbalten: das Chlorzink ist auf den Hydratgehalt des Kobaltchlorürs ohne Wirkung, das Chlornatrium entzieht ihm das Wasser, lässt es aber gelöst, während das Chlorcalcium es auch aus dem Wasser verdrängt; eine Steigerung in diesen drei Beispielen lässt sich nicht verkennen. Ich hebe ausserdem noch hervor, dass der Uebergang des einen Hydratgehaltes in den andern nicht sprungweise vor sich geht, sondern allmälig, was schon von verschiedenen Seiten festgestellt wurde, sich übrigens auch durch den allmäligen Uebergang einer Farbe in die andere erweist.

Waren diese Schlüsse richtig gezogen: ist es möglich durch geeignete Behandlung das Moleciil irgend einer Substanz in niedrigprocentiger Lösung in den Zustand überzuführen, den es in hoch. procentigen Verhältnissen einnimmt, so musste dies auch an den biologischen Eigenschaften erkennbar und nachweisbar sein, so musste die niedrigprocentige Lösung mit richtig modificirtem Molectil denselben physiologischen und pathologischen Effect erzielen, wie die entsprechende hochprocentige.

Dies nachzuweisen, sind Desinfectionsversuche ganz besonders geeignet and habe ich deshalb solche vornehmlich am Carbol und den drei Kresolen, später auch an den drei Dihydroxylbenzolen, ferner dem Pyrogallol und dem Tannin angestellt.

Der Uebergang von einem Hydrat in einen andern Hydratgehalt ist bei diesen Substanzen durch Farbänderung natiurlich nicht zu erkennen, dagegen giebt das beginnende Ausfallen dieser Körper, die Trübung der Flüssigkeit ein genügend zuverlässiges Merkmal ab, um rechtzeitig zu bemerken, dass der Hydratgehalt des Molecüls soweit gesunken ist, dass der Körper sich nicht mehr in Lösung zu halten vermag, dass er also auf seiner niedersten Hydratstufe angelangt ist, welche unter den gegebenen Verhältnissen ubberhaupt möglich ist. 
Was das Bestehen von Hydraten bei diesen Substanzen betrifft, so ist beim Carbol die Bildung von solchen durch die Existenz des "Acidum carbolicum liquefactum" jedermann bekannt. Genauere Untersuchungen hieriber liegen von Alexejeff vor.

Er stellte dieselben folgendermaassen an: Zugeschmolzene Glasröhren, welche abgewogene Mengen Phenol und Wasser enthielten, wurden in einem mit Wasser gefullten Becherglas so lange erwärmt, bis der Röhreninhalt klar wurde. Dann wurde unter fortwährendem Umrühren nach und nach kaltes Wasser bis zum Eintritt der Trubung: zugesetzt.

Seine Resultate sind aus folgenden zwei Tabellen ersichtlich:

\section{Löslichkeit von Phenol in Wasser.}

\begin{tabular}{|c|c|c|c|c|c|c|c|c|c|c|c|}
\hline$\%$-Gehalt an Phenol & 3,75 & 4,83 & 4,85 & 5,13 & 5,27 & 5,36 & 6,19 & 7,33 & 8,03 & 9,83 & 11,83 \\
\hline $\begin{array}{l}\text { Temp. bei d. Trubung } \\
\text { eintritt in } \mathrm{C} \text {. }\end{array}$ & $\begin{array}{l}\text { trubt sich } \\
\text { nicht bei } \\
-10^{\circ} \mathrm{C} \text {. }\end{array}$ & +11 & 12 & 25 & 26 & 35 & 45 & 58 & 59 & 72 & 77 \\
\hline
\end{tabular}

\begin{tabular}{l|l|l|l|l|l|l|l|l|l|l|l}
\hline$\%$-Gehalt an Phenol & 13,78 & 17,3 & 17,97 & 18,78 & 20,05 & 23,77 & 24,12 & 33,23 & 37,39 & 40,01 \\
\hline \hline $\begin{array}{c}\text { Temp. bei der Trubung } \\
\text { eintritt in } 0 \text { C. . }\end{array}$ & 80 & 82 & 83 & 83 & 83 & 84 & 84 & 84 & 84 & 84
\end{tabular}

II. Löslichkeit von Wasser in Phenol.

\begin{tabular}{c|c|c|c|c|c|c|c|c}
\hline \%o-Gehalt an Wasser & 16,38 & 19,85 & 20,92 & 23,30 & 26,75 & 26,93 & 31,99 & 34,44 \\
\hline $\begin{array}{c}\text { Temp. bei d. Trubung } \\
\text { eintritt in } 0 \mathrm{C} .\end{array}$ & $\begin{array}{c}\text { trubt sich nicht } \\
\text { bei }-10^{\circ} \mathrm{C} .\end{array}$ & -5 & +9 & +32 & 43 & 53 & 60
\end{tabular}

\begin{tabular}{l|c|c|c|c|c|c|c|c|c}
\hline$\%$-Gehalt an Wasser & 34,77 & 35,10 & 40,72 & 47,54 & 49,52 & 59,85 & 59,99 & 66,77 & 76,23 \\
\hline $\begin{array}{c}\text { Temp. bei d. Trubung } \\
\text { eintritt in } 0 \text { C. }-\end{array}$ & 61 & 62 & 71 & 78 & 80 & 84 & 84 & 84 & 84
\end{tabular}

Das Phenol löst sich also nach Alex ejeff nicht nur in Wasser, sondern das Wasser auch in Phenol oder wie ich mich ausdrticken möchte, das Phenol bildet mit Wasser zwei Reihen von Hydraten. Dieselben vereinigen sich bei $84^{\circ} \mathrm{C}$, von hier an mischt sich Phenol und Wasser in jedem Verhältniss.

Während hier die Wärme als das den Hydratgehalt herabsetzende Moment beniutzt wurde, verwendete ich in den nachstehenden Versuchen als solches in der Hauptsache Kochsalz, dann auch Natron bicarbonicum, Glaubersalz, Natriumthiosulfat u. a. m. 
Es kommt einfach darauf an, solche Körper zu finden und zu verwenden, deren Affinität zu Wasser grösser ist als diejenige der Substanzen, deren Hydratgehalt herabgesetzt werden soll.

Meine Methode war folgende: In eine gewogene Glasstöpselflasche wurden $30 \mathrm{ccm}$ der $\mathrm{zu}$ untersuchenden $1-5$ proc. Lösung gefullt, das Ganze wieder gewogen und nun kleine Mengen englischen Kochsalzes zugegeben und tuichtig geschuittelt, bis eine bleibende Tribung entstand; gleichzeitig wurde die Temperatur bestimmt und durch Eintauchen in warmes Wasser Sorge getragen, dass die Temperatur, bei der die Bestimmung erfolgen sollte, eingehalten wurde. Nach Eintritt der Trübung und einigem Stehenlassen wurden die Gläser wieder gewogen und so die verbrauchte Kochsalzmenge festgestellt. Es zeigte sich bei diesen Versuchen, dass der Eintritt der Trübung nicht immer mit absoluter Schärfe festgestellt werden konnte; man war manchmal im Zweifel, handelte es sich um eine Opalescenz nur oder schon um eine Trübung: es ist deshalb auf die entstandenen Decimalen kein grosser Werth zu legen und theile ich sie nur mit, weil sie einmal bestimmt worden sind.

Die Resultate sind in folgender Tabelle zusammengestellt:

\begin{tabular}{|c|c|c|c|c|c|c|c|c|c|}
\hline \multirow[t]{2}{*}{$\begin{array}{l}\text { Wirksame } \\
\text { Substanz }\end{array}$} & \multirow{2}{*}{ 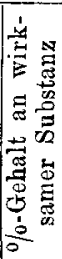 } & \multicolumn{3}{|c|}{$\begin{array}{l}\text { \%o-Gehalt an Koch- } \\
\text { salz, bei dem Trübung } \\
\text { eintritt }\end{array}$} & \multirow[t]{2}{*}{$\begin{array}{l}\text { Wirksane } \\
\text { Substanz }\end{array}$} & \multirow{2}{*}{ 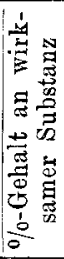 } & \multirow{2}{*}{ 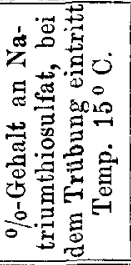 } & \multirow{2}{*}{ 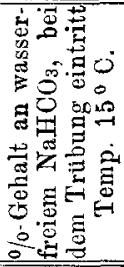 } & \multirow{2}{*}{ 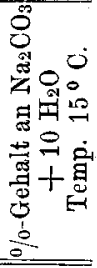 } \\
\hline & & $\begin{array}{l}\text { Temp. } \\
20^{\circ} \mathrm{C} .\end{array}$ & $\begin{array}{l}\text { Temp. } \\
15^{\circ} \mathrm{C} .\end{array}$ & $\begin{array}{l}\text { Temp. } \\
10^{\circ} \mathrm{C} .\end{array}$ & & & & & \\
\hline \multirow{5}{*}{ Carbol } & 5 & 5,57 & 3,5 & - & \multirow{10}{*}{ 0-Kresol } & - & - & - & - \\
\hline & 4 & 10,61 & 7,0 & - & & - & _. & - & - \\
\hline & 3 & 13,53 & 12,0 & $\ldots$ & & - & _. & - & - \\
\hline & 2 & 20,37 & 17,0 & - & & $\ldots$ & - & - & - \\
\hline & 1 & 34,74 & 24,0 & - & & - & - & - & - \\
\hline \multirow{5}{*}{ o-Kresol } & $2^{1 / 2}$ & 0,5 & - & -- & & $21 / 2$ & - & 9,00 & 1,0 \\
\hline & 2 & 2,96 & 3,00 & 2,92 & & 2 & 6,27 & 12,00 & 8,0 \\
\hline & $11 / 2$ & 8,27 & 6,64 & 5,98 & & $11 / 2$ & 13,07 & 16,00 & 17,0 \\
\hline & 1 & 13,43 & 12,62 & 12,62 & & 1 & 25,82 & 23,60 & 32,0 \\
\hline & $1 / 2$ & 25,65 & 21,85 & 20,86 & & $1 / 2$ & 48,88 & 34,00 & 66,0 \\
\hline \multirow{4}{*}{ m-Kresol } & 2 & 2,32 & - & - & \multirow{4}{*}{ m-Kresol } & 2 & 2,6 & - & - \\
\hline & $1 \frac{1}{2}$ & 6,16 & - & - & & $11 / 2$ & 7,92 & & \\
\hline & 1 & 11,36 & - & - & & 1 & 21,43 & - & - \\
\hline & $1 / 2$ & 23,93 & - & 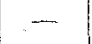 & & $1 / 2$ & 42,76 & 一 & - \\
\hline \multirow{4}{*}{ p-Kresol } & 2 & 1,65 & 1,67 & 1,31 & \multirow{4}{*}{ p-Kresol } & 2 & 0,99 & - & - \\
\hline & $11 / 2$ & 6,27 & 4,90 & 4,25 & & $11 / 2$ & 7,23 & - & - \\
\hline & 1 & 10,67 & 10,21 & 9,04 & & 1 & 19,60 & - & - \\
\hline & $1 / 2$ & 21,47 & 20,26 & 19,28 & & $1 / 2$ & 41,50 & - & - \\
\hline
\end{tabular}




\begin{tabular}{|c|c|c|c|c|c|c|c|c|c|}
\hline \multirow{2}{*}{$\begin{array}{l}\text { Wirksame } \\
\text { Substanz }\end{array}$} & \multirow{2}{*}{ 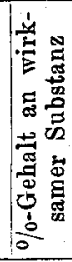 } & \multicolumn{3}{|c|}{$\begin{array}{l}\% \text {-Gehalt an Koch- } \\
\text { salz, bei dem Trubung } \\
\text { eintritt }\end{array}$} & \multirow{2}{*}{$\begin{array}{l}\text { Wirksame } \\
\text { Substanz }\end{array}$} & \multirow{2}{*}{ 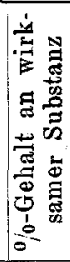 } & \multirow{2}{*}{ 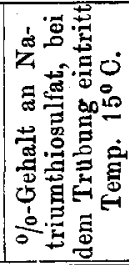 } & \multirow{2}{*}{ 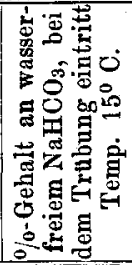 } & \multirow{2}{*}{ 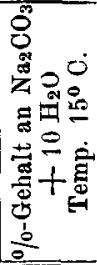 } \\
\hline & & $\begin{array}{l}\text { Temp. } \\
20^{\circ} \mathrm{C} .\end{array}$ & $\begin{array}{l}\text { Temp. } \\
15^{\circ} \mathrm{C} .\end{array}$ & $\begin{array}{l}\text { Temp. } \\
10^{0} \mathrm{C} .\end{array}$ & & & & & \\
\hline $\begin{array}{c}\text { Brenz- } \\
\text { katechin }\end{array}$ & $\begin{array}{l}5 \\
4 \\
3 \\
2 \\
1\end{array}$ & $\begin{array}{l}\mathbf{3 3 , 2 2} \\
\mathbf{3 4 , 0 7} \\
\mathbf{3 5 , 1 9} \\
\mathbf{3 6 , 5 1} \\
\mathbf{3 7 , 5 8}\end{array}$ & \multicolumn{2}{|c|}{$\begin{array}{l}\text { Bei Deberschuss } \\
\text { v. Kochsalz blieb } \\
\text { dieses ungelöst; } \\
\text { Brenzkatechin } \\
\text { fiel nicht aus }\end{array}$} & 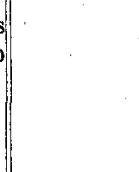 & $\begin{array}{l}- \\
\overline{-} \\
-\end{array}$ & $\begin{array}{c}- \\
- \\
-\end{array}$ & $\begin{array}{l}- \\
\overline{-} \\
-\end{array}$ & $\begin{array}{l}- \\
- \\
-\end{array}$ \\
\hline Resorcin & $\begin{array}{l}5 \\
4 \\
3 \\
2 \\
1\end{array}$ & $\begin{array}{l}24,21 \\
26,88 \\
27,97 \\
29,84 \\
33,22\end{array}$ & $\begin{array}{l}- \\
- \\
- \\
-\end{array}$ & $\begin{array}{l}- \\
\overline{-} \\
-\end{array}$ & & $\begin{array}{l}\bar{z} \\
\overline{-}\end{array}$ & $\begin{array}{l}- \\
z \\
-\end{array}$ & $\begin{array}{l}\bar{z} \\
\bar{z}\end{array}$ & $\frac{-}{-}$ \\
\hline $\begin{array}{l}\text { Hydro- } \\
\text { chinon }\end{array}$ & $\begin{array}{l}5 \\
4 \\
3 \\
2 \\
1\end{array}$ & $\begin{array}{r}6,73 \\
10,64 \\
14,37 \\
24,03 \\
31,19\end{array}$ & $\begin{array}{l}- \\
- \\
-\end{array}$ & $\begin{array}{l}- \\
- \\
-\end{array}$ & & $\begin{array}{l}- \\
\overline{-}\end{array}$ & $\begin{array}{l}- \\
\bar{z} \\
-\end{array}$ & $\begin{array}{l}- \\
- \\
-\end{array}$ & $\begin{array}{l}- \\
- \\
-\end{array}$ \\
\hline Pyrogallol & $\begin{array}{l}5 \\
4 \\
3 \\
2 \\
1\end{array}$ & $\begin{array}{l}31,21 \\
32,90 \\
35,50 \\
37,08 \\
37,33\end{array}$ & $\begin{array}{l}\text { Bei Ue } \\
\text { v. Koch } \\
\text { dieses t } \\
\text { Pyrog } \\
\text { niol }\end{array}$ & $\begin{array}{l}\text { erschuss } \\
\text { salz blieb } \\
\text { ngelöst; } \\
\text { allol fiel } \\
\text { t aus }\end{array}$ & & $\begin{array}{l}- \\
- \\
-\end{array}$ & $\begin{array}{l}- \\
- \\
-\end{array}$ & $\begin{array}{l}- \\
= \\
-\end{array}$ & $\begin{array}{l}- \\
- \\
-\end{array}$ \\
\hline Tannin & $\begin{array}{l}5 \\
4 \\
3 \\
2 \\
1\end{array}$ & $\begin{array}{l}10,64 \\
11,36 \\
13,27 \\
15,68 \\
16,55\end{array}$ & $\begin{array}{l}- \\
\overline{-} \\
-\end{array}$ & $\overline{-}$ & & $\begin{array}{l}\overline{-} \\
\overline{-}\end{array}$ & $\begin{array}{l}- \\
z \\
-\end{array}$ & $\begin{array}{l}- \\
z \\
-\end{array}$ & $\begin{array}{l}- \\
\overline{-} \\
-\end{array}$ \\
\hline
\end{tabular}

Wie man sieht, wurde die Hauptsache der Versuche bei $20^{\circ} \mathrm{C}$. angestellt; bei $15^{\circ} \mathrm{C}$. waren für Carbol deutlich geringere Mengen zur Ausfällung nöthig; bei den Kresolen dagegen war der Unterschied, wenn auch meist vorhanden, doch kaum nennenswerth. Für Brenzcatechin und Pyrogallol erwies sich das Kochsalz zum Ausfällen nicht geeignet.

Nach Feststellung dieser Resultate konnte ich zu den Desinfectionsversuchen tibergehen. Stets wurde so verfahren, dass zu $50 \mathrm{ccm}$ der Lösung $1 \mathrm{ccm}$ einer Bacterienaufschwemmung zugefügt wurde und dann aus dieser Suspension nach bestimmter Zeit eine Oese entnommen und in Gelatine oder Agar übertragen wurde.

Zuerst hatte ich den Desinfectionswerth schwacher antiseptischer Lösungen festzustellen: ich gebrauchte hierzu zunächst als Testobject den Prodigiosus. Die Prodigiosusaufschwemmung wurde aus $3-5$ 
Agarkulturen und etwa $50 \mathrm{ccm}$ sterilen Wassers bereitet; als Nährboden verwendete ich Gelatine. Von Desinfectionsflüssigkeiten wurden untersucht:

1. Acid. carbol. cryst. . . . . . 1,0:100,0

2. Acid. carbol. liquef. . . . . 1,0:100,0

3. 0 -Kresol. cryst. . . . . . 1,0 10 100,0

4. 0 -Kresol. pur. liquef. Nördlinger $1,0: 100,0$

5. m-Kresol. pur. . . . . . . . 1,0:100,0.

Nach 15, 30 und 60 Secunden wurden Platten gegossen:

1. Acid. carb. cryst. zeigte nach 1 Minute noch 2 Colonien.

2. Acid. carb. liq. nach 1 Minute noch 1000 Colonien.

3. o-Kresol. cryst. nach $1 / 4$ Minute 37 Colonien, nach $1 / 2$ Minute 0 Colonien.

4. o-Kresol. pur. liq. nach 1/4 Minute 200 Colonien, nach 1/2 Minute 0 Colonien.

5. m-Kresol. pur. nach $1 / 4$ Minute 35 Colonien, nach $1 / 2$ Minute 0 Colonien.

Die Controllplatte, die genau ebenso hergestellt worden war, nur dass $50 \mathrm{ccm}$ Wasser statt antiseptischer Lösung verwendet wurde, zeigte 5500 Colonien.

Darnach erwies sich der Prodigiosus als ein sehr wenig widerstandsfähiger Mikroorganismus und nicht geeignet, die eventuelle Verstärkung der Desinficientien durch indifferente Zusätze deutlich hervortreten zu lassen.

Deshalb stellte ich genau denselben Versuch mit einem hochvirulenten Staphylococcus pyogenes aureus an, der frisch aus einem Fall von Pyämie geziichtet worden war. Als Nährboden wurde Agar verwendet; die Platten standen bei $37^{\circ} \mathrm{C}$.

Am 5. Tag ergab die Controlplatte $64800 \mathrm{Keime,}$ die übrigen Platten zeigten folgendes Ergebniss:

\begin{tabular}{l|r|r|r|r|r|r}
\hline 1 Proc. & $\begin{array}{r}\text { nach } \\
\text { 1 Min. }\end{array}$ & $\begin{array}{c}\text { nach } \\
\text { 2 Min. }\end{array}$ & $\begin{array}{c}\text { nach } \\
\text { 3 Min. }\end{array}$ & $\begin{array}{r}\text { nach } \\
\text { 4 Min. }\end{array}$ & $\begin{array}{r}\text { nach } \\
5 \text { Min. }\end{array}$ \\
\hline \hline 1. Acid. carb. cryst. & 32400 & 27600 & 18900 & 17500 & 14570 \\
2. Acid. carb. liq. & 42850 & 36750 & 28900 & 19450 & 17700 \\
3. o-Kres. pur. oryst. & 16800 & 15000 & 14200 & 10800 & 5400 \\
4. o-Kres. pur. liq. . & 30000 & 24000 & 18900 & 16800 & 6100 \\
5. m-Kres. pur. . . & 10800 & 8100 & 2620 & 1.230 & 814
\end{tabular}

Also keines der genannten Desinfectionsmittel zerstörte in 1 proc. Lösung meinen Staphylococcus aureus innerhalb 5 Minuten.

Nun setzte ich zu diesen Lösungen die oben gefundene Kochsalz- 
menge und untersuchte ausserdem die concentrirten Lösungen dieser Substanzen; sonach hatte ich folgende Recepte:

1. Acid. carbol. cryst. 1,0:100,0, Kuchsalz 24,0.

2. Acid. carb. eryst. $6,0: 100,0$.

3. o-Kresol. cryst. 1,0:100,0, Kochsalz 13,0.

4. 0 -Kresol. $2,5: 1,00$.

5. m-Kresol. 1,0:100,0, Kochsalz 12,0.

6. m-Kresol. $2,5: 100,0$.

7. Kochsalz 24,0:100,0 als Controle.

Das Resultat des in der sonstigen Versuchsanordnung mit dem vorherigen genau übereinstimmenden, auch am selben Tage ausgeführten Versuches veranschaulicht folgende Tabelle:

\begin{tabular}{|c|c|c|c|c|c|c|c|}
\hline $\begin{array}{l}\text { Zeitdauer } \\
\text { der Ein- } \\
\text { wirkung }\end{array}$ & $\begin{array}{c}\text { Carbol } \\
1: 100, \\
\text { Kochsalz } \\
24 .\end{array}$ & 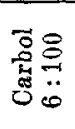 & $\begin{array}{c}\text { o-Kres. } \\
1: 100 \\
\text { Kochsalz } \\
13 .\end{array}$ & 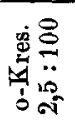 & $\begin{array}{c}\text { m-Kres. } \\
1: 100 \\
\text { Kochsalz } \\
12 .\end{array}$ & 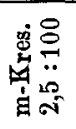 & $\begin{array}{l}\text { Kochsalz } \\
24: 100\end{array}$ \\
\hline $\begin{aligned} 1 / 4 & \text { Minute } \\
1 / 2 & \text { Minute } \\
1 & \text { Minute }\end{aligned}$ & $\begin{array}{l}8 \\
0 \\
0\end{array}$ & $\begin{array}{l}2 \\
0 \\
0\end{array}$ & $\begin{array}{l}7 \\
2 \\
0\end{array}$ & $\begin{array}{l}0 \\
0 \\
0\end{array}$ & $\begin{array}{l}3 \\
1 \\
0\end{array}$ & $\begin{array}{l}1 \\
0 \\
0\end{array}$ & $\begin{array}{l}45000 \\
46200 \\
37800\end{array}$ \\
\hline
\end{tabular}

Nach einer halben Minute hatten also diese einprocentigen PhenolKochsalzlösungen alles vernichtet, ganz ebenso wie die concentrirten Lösungen.

Nun war für mich nur noch festzustellen, wie lange die einprocentigen Phenollösungen ohne Zusatz uiberhaupt brauchen, den Staphylococcus aureus zu tödten; ich stellte eine dahinzielende Versuchsreihe mit 1 proc. Lösungen von Carbol cryst. und o-Kresol cryst. an; es zeigte sich, dass erstere hierzu 20 Minuten brauchte, letztere 7 Minuten. Die Resistenz des Staphylococcus aureus nimmt übrigens bei Züchtung auf künstlichen Nährböden deutlich ab; als ich einen Monat später den Versuch wiederholte mit einer von demselben Aureus stammenden 6 tägigen Agarkultur, brauchte die 1 proc. Carbollösung nur noch 15, die 1 proc. o-Kresollösung nur noch 5 Minuten zur Abtödtung desselben.

Das ausgezeichnete Resultat meiner Versuche mit Phenol-Kochsalzlösungen veranlasste mich, auch deren Wirkung auf Milzbrandsporen in den Kreis meiner Beobachtung zu ziehen. Die mit diesem Testobject untersuchten Flüssigkeiten waren folgende:

1. 1 pro mille Sublimat.

2. 1 Proc. Carbol cryst.

3. 1 Carbol +24 Proc. Kochsalz.

4. $3=$ Carbol.

5. 3 Carbol +12 Proc. Kochsalz. 
6. 6 Proc. Carbol.

7. $1 / 2=0$-Kresol +19 Proc. Kochsalz.

8. $1 \Rightarrow$-Kresol.

9. $1=0$-Kresol +13 Proc. Kochsalz.

10. $2,5=0$-Kresol.

$\left.\begin{array}{l}\text { 11. } 24=\text { Kochsalz } \\ \text { 12. Aq. dest. steril. }\end{array}\right\}$ als Controle.

Wie in den früheren Versuchen, wurden Agarplatten gegossen und diese bei Bruttemperatur gehalten.

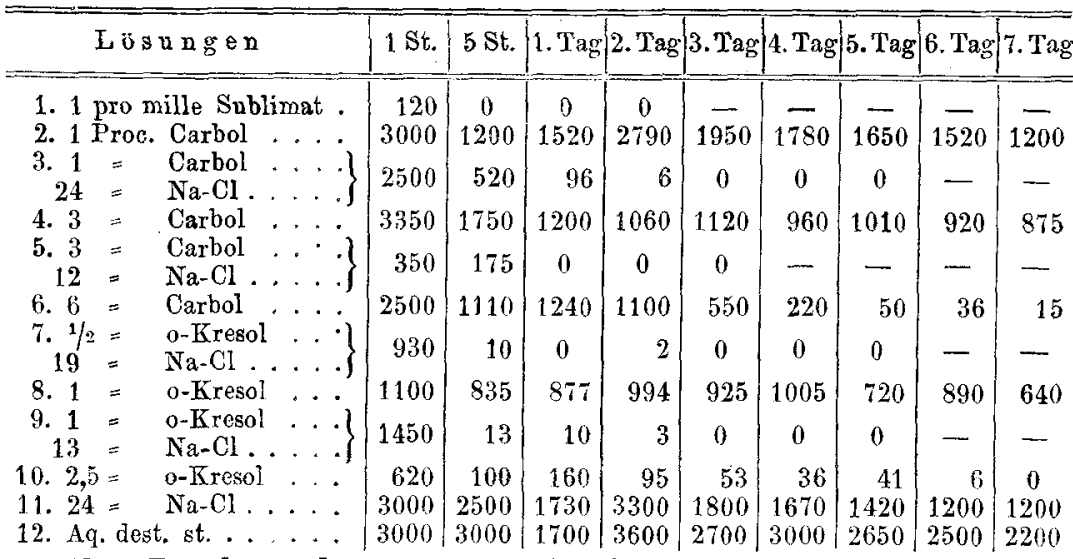

Das Resultat, das aus vorstehender Tabelle erhellt, war ein ganz ïberraschendes: das 1 proc. und 3 proc. Carbol, das $1 / 2$ proc. wie 1 proc. o-Kresol hatte, mit dem entsprechenden Kochsalzzusatz versehen, Milzbrandsporen in spätestens 3 Tagen vernichtet, während ohne Kochsalz kaum eine Einwirkung auf die Milzbrandsporen zu erkennen war.

Einen zweiten Milzblandsporenversuch begann ich am 20. August 1894. Doch ist hervorzuheben, dass jetzt andere Verhältnisse vorlagen, als beim ersten; während zur Zeit, als dieser angestellt wurde - Beginn am 27. Juni und wiederholt am 3. Juli 1894 - es sehr heiss war und das Thermometer über Mittag $23^{\circ} \mathrm{R}$. zeigte, war es Ende August kalt und die höchste Temperatur im Laboratorium betrug $14^{0} \mathbf{R}$. Dieser Umstand konnte bei der mehrfach erwähnten Bedeutung der Wärme für den Molecularzustand der in Rede stehenden Substanzen nicht ohne Einfluss auf die Desinfectionswirkung bleiben und sehen wir in Folge dessen eine wenn auch geringe, so doch deutliche Abschwächung derselben in diesem Versuch gegenüber dem ersten eintreten. 
Die untersuchten Lösungen waren folgende:

1. Acid. carbol. cryst. 2 Proc., Kochsalz 20 Proc.

2. o-Kresol. pur. liq. 1 Proc., Kochsalz 12 Proc.

3. m-Kresol 1 Proc., Kochsalz 12 Proc.

4. p-Kresol 1 Proc., Kochsalz 10 Proc.

5. Resorcin 2 Proc., Kochsalz 30 Proc.

6. Pyrogallol 2 Proc., Kochsalz 35 Proc.

7. Tannin 2 Proc., Kochsalz 15 Proc.

8. Brenzcatechin 2 Proc., Kochsalz 35 Proc.

9. Hydrochinon 2 Proc., Kochsalz 25 Proc.

10. Formalin 5 Proc., Kochsalz 12 Proc.

11. Sublimat 1 pro mille.

12. Wasser.

Es schieden sich, da die angewandte Kochsalzmenge meist die fur $20^{\circ} \mathrm{C}$. bestimmte war, die Fliussigkeit in diesem Versuch aber nur $12^{\circ} \mathrm{C}$. hatte, einige Körper aus: die 3 Kresole hatten eine deutliche Fettropfenschicht uud das Tannin war getrübt. Diese Lösungen wurden filtrirt und dann verwendet; dass sie nun natürlich nicht mehr genau den angegebenen Werthen entsprachen, ist mir wohlbekannt, doch war der Unterschied nicht gerade gross. Bei der Gelegenheit hebe ich hervor, dass ich während dieser ganzen Arbeit Sorge trug, dass nie eine Fliussigkeit zum Versuch gelangte, in der Ausscheidungen vorbanden gewesen wären.

Dem Formalin habe ich ganz obne jeden sonstigen Anhalt 12 Proc. Kochsalz zugesetzt; es traten bald an der Glaswand Gasblasen auf und der verstärkte stechende Geruch bewies eine vermehrte Verdunstung.

Die nicht besonders erwähnten Lösungen in diesem letzten Versuch waren von vorn herein klar; das Ergebniss desselben erhellt aus nachfolgender Tabelle:

\begin{tabular}{|c|c|c|c|c|c|c|c|c|c|c|c|}
\hline \multicolumn{3}{|c|}{ Lo s ungen } & $1 / 2 \mathrm{St}$. & 1. Tag & 2. Tag & 3. Tag & 4. Tag & 5. Tag & 6. Tag & 7. Tag & 8. Tag \\
\hline 1. $\begin{array}{r}2 \\
20\end{array}$ & $\begin{array}{l}\text { Proc. } \\
=\end{array}$ & $\begin{array}{l}\text { Carbol } \ldots \ldots \\
\mathrm{Ca}-\mathrm{Cl} \ldots\end{array}$ & 4200 & 1560 & 540 & 120 & 0 & 0 & - & - & - \\
\hline 2. $\begin{array}{r}1 \\
12\end{array}$ & $=$ & $\left.\begin{array}{l}\mathrm{o}-\mathrm{Kr} r e s o l \\
\mathrm{Na}=\mathrm{Cl} \ldots\end{array} \ldots.\right\}$ & 3600 & 1620 & 850 & 250 & 40 & 0 & 0 & - & - \\
\hline 3. $\begin{array}{r}1 \\
12\end{array}$ & & $\begin{array}{l}\mathrm{m} \text {-Kresol } \\
\mathrm{Na}-\mathrm{Cl} . .\end{array}$ & 5800 & 1870 & 600 & 320 & 75 & 0 & 0 & - & $一$ \\
\hline 4. $\begin{array}{r}1 \\
10\end{array}$ & $=$ & $\begin{array}{l}\text { p-Kresol . } \\
\text { Na-Cl . . }\end{array}$ & 7500 & 860 & 500 & 180 & 130 & 90 & 0 & 0 & - \\
\hline 5. $\begin{array}{r}2 \\
30\end{array}$ & $\begin{array}{l}= \\
=\end{array}$ & $\begin{array}{l}\text { Resorcin .... } \\
\mathrm{Na}-\mathrm{Cl} . . .\end{array}$ & 3500 & 3840 & 3280 & 2950 & 2640 & 2040 & 1780 & 970 & 275 \\
\hline 6. $\begin{array}{r}2 \\
35\end{array}$ & $\begin{array}{l}= \\
=\end{array}$ & $\begin{array}{l}\text { Pyrogallol ... } \\
\text { Na-Cl ..... }\end{array}$ & 4500 & 2870 & 2540 & 420 & 380 & 290 & 160 & 70 & 60 \\
\hline
\end{tabular}




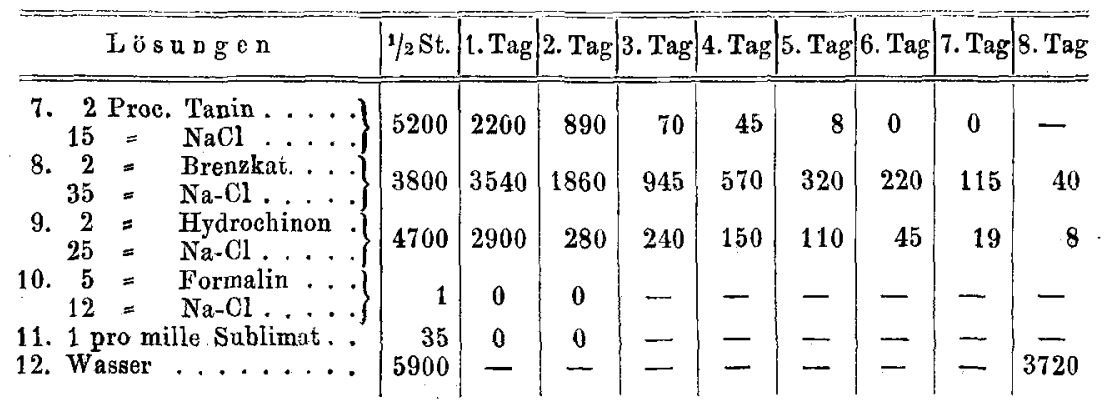

In Wesentlichen stimmt also das Resultat dieses Versuches mit dem früheren überein. Hervorzuheben ist der ganz bedeutende Desinfectionswerth des Tannins, besonders deshalb, da ausser dem Kochsalz noch eine Reihe von anderen indifferenten Substanzen existiren, die das Tannin in seinen Lösungen ebenso beeinflussen wie dieses; hier können weitere Versuche zu direct praktischen Erfolgen führen.

Hiermit schliessen meine Versuche. Durch äussere Umstände bin ich am Weiterarbeiten verhindert worden.

Wir haben also gesehen, dass die Phenole mit Wasser Hydrate bilden und dass der Hydratgehalt bei schwachen Lösungen ein grös* serer, bei concentrirten ein geringerer ist. Diese Hydrate geben beim Erhitzen ihr Wasser allmählich ab. Ganz ähnlich kann es ihnen in grösserem oder geringerem Grade, meist je nach der Versuchstemperatur, durch andere stärker wasserbindende Körper entzogen werden. - Beim Carbol und den Kresolen wird das Hydrat der einen Reihe schliesslich in das der anderen gebracht und fällt aus. -

So kann das Carbolmolecül einer 1 proc. Lösung durch Kochsalz, Glaubersalz und andere Zusätze in denselben Zustand gebracht werden wie das Molecül einer gesättigten Lösung, also bei Zimmertemperatur einer 6-7 proe. Beide Molecüle sind sich dann also gleich und demgemäss auch ihr dynamischer Werth. Eine solche 1 proc. Lösung hat somit denselben Desinfectionswerth wie eine concentrirte. Dass ich, gleicbgilltig, ob mit einer halb-, ein-, zwei- oder dreiprocentigen Lösung unter Zusatz der vorher bestimmten nothwendigen Salzmenge experimentirend stets den gleich hohen Desinfectionswerth erzielte, dieser Umstand lässt es zweifellos erscheinen, dass hier ein Naturgesetz obwaltet.

Aus diesen Versuchen geht ferner hervor, dass die Angabe des Procentgehalts einer Fliussigkeit an wirksamer Substanz uns uber den Wirkungswerth derselben vollständig im Unklaren lassen kann, dieselbe darf nur darin vergesellschaftet sein mit einer Substanz, die, 
wie in unseren Fällen, zu Wasser eine grössere Affinität besitzt als diese selbst.

Daraus folgt weiter, dass wir in der biologischen Chemie mindestens eben so sehr mit einer Affinität der Molecüle, als mit einer solchen der Atome zu rechnen haben, deren Valenz, wie aus dem Beispiel des Zinkchlorids, verglichen mit dem Kochsalz, hervorgeht, ebenso verschieden ist, wie die der Atome.

Ich bin bis jetzt nicht in der Lage gewesen, von der Feststellung dieses Gesetzes zur praktischen Anwendung überzugehen; ich glaube aber, dass sich hier, abgesehen von einem nenen Gesichtspunkt für die verschiedensten Verhältnisse, ein weites Feld in Wissenschaft und Technik darbietet, selbstrerständlich nicht für die Desinfectionspraxis allein, in der bekanntlich rein empirisch ab und za schon die Bemerkung gemacht wurde, dass zusammengesetzte Desinfectionsmittel eine stärkere Wirkung haben, als ibre Komponenten. Ich will nur als Beispiele erwähnen, dass die Wirkung der Ehrlich'schen und Ziehl'schen Carbolfuchsinlösung, die der natürlichen Mineralwässer und noch manches andere durch dieses Gesetz ihre Erklärung finden dïrfte.

Ich habe einigen mir befreundeten Chirurgen die Anwendung der 1 proc. o-Kresollösung mit 12 proc. Kochsalz empfohlen und allgemein hat dieselbe zufrieden gestellt. Uebrigens beginnen die Instrumente schon bei nicht sehr langem Verweilen in dieser Kochsalzlösung zu rosten, ein Umstand, der durch Zusatz von 1 pro Mille Natriumthiosulfat, das reducirend wirkt, leicht verhindert werden kann.

Wichtigere Resultate als für die Chirurgie und Geburtshülfe, dürfte für die Pharmakologie und innere Medicin die Erforschung dieses Gesetzes zeitigen. Man ist weiterbin versucht, eine Theorie der Immunität und Heilung aufzustellen, deren Construction ziemlich einfach ist. Das geht nämlich aus der Verschiedenheit der Aussalzbarkeit der einzelnen Eiweisskörper hervor, dass dieses Gesetz auch für sie Geltung hat, dass sie unter sich selbst eine verschiedene, und zwar eine sebr starke Affinität zu Wasser baben, und dass sie sich somit gegenseitig zu beeinflussen vermögen.

Ich stelle mir bis auf Weiteres auf Grund dieses Gesetzes die künstliche und die natürliche Immunität so vor, dass ich annehme, dass das Heilserum bezw. bei activer Immunisirung die mehr oder weniger veränderten Producte der Bacterien die Stelle des Kochsalzes in unseren Versuchen und die Alexine diejenige der Carbolsäure tibernehmen, während bei der natürlichen Immunität der Molecularzustand 
dieser Alexine entweder durch grössere Concentration oder durch die Wirkung anderer Eiweisskörper in wirksamer Verfassung erhalten wird. Bei dieser Denkweise können wir auch - ich glaube zum ersten Mal - uns eine physikalisch-chemische Vorstellung davon machen, in welcher Art die vielumstrittene und doch zweifellos zu Recht bestehende Wirkung einer Erkältung als begtunstigendes Moment zur Entstehung einer Infectionskrankheit zu Stande kommen kann. 